\title{
Mid-long-term results of total knee arthroplasty followed by ipsilateral total hip arthroplasty versus total hip arthroplasty subsequent to ipsilateral total knee arthroplasty: a case-control analysis
}

\author{
Zunhan Liư ${ }^{\dagger}$, Wei-Nan Zeng ${ }^{\dagger}$, Zhenyu Luo, Enze Zhao, Hao Li and Zongke Zhou* (D)
}

\begin{abstract}
Background: The aim of the present study was to compare the outcomes of patients who underwent different sequences of ipsilateral total hip arthroplasty (THA) and total knee arthroplasty (TKA).

Methods: We retrospectively identified 47 patients who underwent TKA followed by ipsilateral THA (THA-TKA) and 36 patients who received THA subsequent to ipsilateral TKA (TKA-THA) for rheumatoid arthritis or osteoarthritis between January 2008 and April 2014. Twenty-eight patients were selected for each group after case-control matching with preoperative demographics and protheses of THA. Clinical scores, radiographic results, complication rates, and survivorship were compared. The median duration of follow-up was 110 (range 80-149) months.

Results: Both groups showed significant improvement in Harris Hip Scores, Knee Society Score, and Short Form-12 at the last follow-up compared to baseline $(p<.001)$. At the last follow-up, all clinical scores were actually lower in the THA-TKA group, but those differences were not statistically significant. Otherwise, there was no significant difference in radiological alignment or complication rates. The survivorship of THA and TKA in the THA-TKA group was 94.7 and 95.7\%, respectively, compared with 92.4 and 100.0\% in the TKA-THA group at 8 years (log rank, $p=.939$ and .187)
\end{abstract}

Conclusions: Patients who underwent ipsilateral THA and TKA with different sequences achieved similar favorable outcomes. Total joint arthroplasty can be performed safely with excellent outcomes in patients with a history of prior ipsilateral THA or TKA.

Trial registration: The trial was registered in the Chinese Clinical Trial Registry (ChiCTR2000035147) dated 2 August 2020.

Keywords: Total knee arthroplasty, Total hip arthroplasty, Ipsilateral, Mechanical axis, Valgus

\footnotetext{
* Correspondence: zhouzongke@scu.edu.cn

${ }^{\dagger}$ Zunhan Liu and Wei-Nan Zeng contributed equally to this work.

Department of Orthopedic Surgery, West China Hospital, West China Medical

School, Sichuan University, \#37 Guoxue Road, Chengdu, Sichuan Province

610041, People's Republic of China
}

C C The Author(s). 2021 Open Access This article is licensed under a Creative Commons Attribution 4.0 International License, which permits use, sharing, adaptation, distribution and reproduction in any medium or format, as long as you give appropriate credit to the original author(s) and the source, provide a link to the Creative Commons licence, and indicate if changes were made. The images or other third party material in this article are included in the article's Creative Commons licence, unless indicated otherwise in a credit line to the material. If material is not included in the article's Creative Commons licence and your intended use is not permitted by statutory regulation or exceeds the permitted use, you will need to obtain permission directly from the copyright holder. To view a copy of this licence, visit http://creativecommons.org/licenses/by/4.0/. The Creative Commons Public Domain Dedication waiver (http://creativecommons.org/publicdomain/zero/1.0/) applies to the data made available in this article, unless otherwise stated in a credit line to the data. 


\section{Background}

Although the utilization of disease-modifying medications has improved the quality of life of patients with symptomatic arthritis, the number of people affected with multiple lower extremity joints remains high $[1,2]$. Subsequently, the possibility of performing total joint arthroplasty (TJA) on the ipsilateral hip and knee in the same patient may increase. A previous study reported that the cumulative incidences of total knee arthroplasty (TKA) followed by ipsilateral total hip arthroplasty (THA) and THA subsequent to ipsilateral TKA were 2.1 and $1.8 \%$, respectively, at the 20-year follow-up [3].

TJA is one of the most successful health care interventions. Patients with end-stage degenerative or inflammatory arthritis report excellent outcomes after THA or TKA [4]. However, there is some evidence to suggest loading redistribution and biomechanical changes in the lower limb after THA or TKA [5-9]. Data concerning the influence of prior THA on the ipsilateral knee joint or ipsilateral TKA remain controversial. Several studies have reported that the lateral patellar tilt increased and the external knee adduction moment decreased after ipsilateral THA, which may lead to abnormal loading on the knees and ipsilateral knee pain $[5,7,8]$. Other studies have suggested that there is no increase in biomechanical loading during gait and posture on ipsilateral or contralateral knee joints after unilateral THA [10]. Furthermore, in certain cases, it is difficult to thoroughly insert the femoral intramedullary guide into the femoral canal during ipsilateral TKA due to a prior femoral prosthesis, increasing the chance of malalignment [11]. To date, only one study has analyzed the influence of prior THA on the functional outcome of subsequent ipsilateral TKA, which demonstrated the influence is limited [12]. On the other hand, the influence of a prior TKA on the ipsilateral hip has also been examined. Previous studies have reported that prior TKA leads to biomechanical changes and that knee alignment caused by prior TKA affects the positioning of the ipsilateral femoral component during THA [6, 13-15]. However, to our knowledge, no study has yet compared the clinical outcomes and implant survivorships in patients undergoing different sequences of ipsilateral THA and TKA.

Therefore, the primary purpose of this study was to determine whether the different sequences of ipsilateral THA and TKA would have different clinical scores. As a secondary outcome, we examined whether the different sequences of ipsilateral THA and TKA would lead to different radiological alignments or component positions, complication rates, and implant survivorships. We hypothesized that patients undergoing different sequences of ipsilateral THA and TKA would have similar clinical outcomes, complication rates, and implant survivorship at the last follow-up.

\section{Methods}

\section{Patients}

This retrospective study was approved by the Institutional Review Board of West China Hospital (ID: 2012268) and was reported in accordance with the STROCSS criteria [16]. The inclusion criteria were patients with severe pain and/or considerable difficulty in performing daily activities refractory to nonoperative management who underwent ipsilateral THA and TKA for rheumatoid arthritis (RA) or osteoarthritis (OA). The exclusion criteria were hip dysplasia, acetabulum or femoral fracture, ankylosing spondylitis, prior lower extremity fracture, posttraumatic arthritis, revision THA or TKA, and incomplete clinical or radiographic records. Additionally, to avoid bias related to prior TJA, patients with an interval time between ipsilateral THA and TKA shorter than 6 months and patients who underwent prior TJA with a functional score at the time of subsequent surgery less than 70 points were also excluded. We identified 88 patients (97 hips and 97 knees) who underwent ipsilateral THA and TKA at our institution between January 2008 and April 2014. Of these, two patients (2 hips and 2 knees) were lost to follow-up and could not be contacted via telephone, while three patients ( 4 hips and 4 knees) died from diseases unrelated to the operation. According to different sequences of ipsilateral THA and TKA, the remaining 83 patients (91 hips and 91 knees), including 47 patients undergoing TKA followed by ipsilateral THA and 36 as THA subsequent to ipsilateral TKA, were classified into 2 groups. To minimize possible confounding factors, the two groups were statistically matched for age (up to \pm 10 years), sex, cause of TJA, primary surgery data (up to \pm 12 months), and prosthesis of THA by utilizing case-control matching at a 1:1 ratio. Because all implants of primary TKA in our center were posteriorstabilized, we did not match the prosthesis of TKA. Ultimately, 28 patients were selected for each group and included in the final analysis. Table 1 shows and compares demographic characteristics between the 2 groups. Case-control matching was conducted using SPSS statistical software, version 25.0 (IBM Corp., Armonk, NY).

\section{Surgical techniques}

All THA procedures were performed using a posterolateral approach under general anesthesia. The cementless porous-coat acetabular fixations (Pinnacle implants, DePuy Orthopaedics) were routinely press-fitted into the acetabulum at $15 \pm 10^{\circ}$ of anteversion and $40 \pm 10^{\circ}$ of inclination. If necessary, supplemental screws were used to achieve implant stability. Two cementless femoral stems, Corail and Summit (DePuy Synthes, Warsaw, IN), were inserted into the hips. Of these, ceramic-on-ceramic articulations were utilized in 42 hips (75.0\%), and ceramicon-polyethylene was used in 14 hips $(25.0 \%)$. 
Table 1 Baseline characteristics of the 2 case control groups

\begin{tabular}{|c|c|c|c|}
\hline Parameters & $\begin{array}{l}\text { THA-TKA group } \\
n=28\end{array}$ & $\begin{array}{l}\text { TKA-THA group } \\
n=28\end{array}$ & $\begin{array}{l}P \\
\text { value }\end{array}$ \\
\hline Gender (male/female) & $3 / 25$ & $3 / 25$ & $1.000^{c}$ \\
\hline Age (yr) & $57.3 \pm 10.6$ & $58.8 \pm 10.2$ & $0.610^{\mathrm{a}}$ \\
\hline Body mass index $\left(\mathrm{kg} / \mathrm{m}^{2}\right)$ & $22.3(20.2-24.9)$ & $22.2(19.4-24.8)$ & $0.928^{b}$ \\
\hline ASA $(I-I /|I I|-\mid V)$ & $19 / 9$ & $22 / 6$ & $0.547^{c}$ \\
\hline No. of diseases (RA/OA) & $17 / 11$ & $17 / 11$ & $1.000^{c}$ \\
\hline Varus/neutral/valgus/severe valgus & $2 / 11 / 14 / 1$ & $3 / 6 / 17 / 2$ & $0.591^{c}$ \\
\hline THA follow-up (mo) & 109.0 (100.3 to 120.3$)$ & 97.5 (82.3 to119.3) & $0.095^{b}$ \\
\hline TKA follow-up (mo) & 100.0 (88.8 to 111.0$)$ & 114.5 (94.3 to 134.3$)$ & $0.012^{\mathrm{b}}$ \\
\hline Interval between surgery (mo) & 11.0 (7.3 to 14.8$)$ & 15.0 (8.3 to 19.5$)$ & $0.061^{b}$ \\
\hline
\end{tabular}

ASA American Society of Anesthesiologists Scale, RA Rheumatoid arthritis, OA Osteoarthritis

${ }^{\text {a }}$ Student $t$ test

${ }^{\mathrm{b}}$ Mann-Whitney $U$ test

'Fisher's exact test

For the ipsilateral TKA procedure, the knee was exposed by a standard medial parapatellar approach, and osteophytes, worn meniscus, and posterior cruciate ligament of the knee were resected. After determining the entry point, we inserted a femoral intramedullary alignment rod in the center of the femoral intercondylar notch with the distal femoral cutting guide set for individual degrees measured before surgery. Of note, one knee in the THA-TKA group and 2 knees in the TKATHA group had a preoperative anatomic valgus of $>20^{\circ}$ that we released the iliotibial band (ITB) using the "piecrusting" technique [17]. If the lateral tension on extension and flexion was still tight, the posterolateral capsule was also released, avoiding the collateral ligament (LCL) and popliteus tendon (POP). After assessing the extension gap and balancing the flexion gap, we performed cemented, posterior-stabilized TKAs on all subjects with two total knee implants (DePuy Sigma PFC and Stryker Scorpio NRG). All patients received intraoperative and postoperative prophylactic broad-spectrum antibiotics and tranexamic acid antithrombotic therapy. Postoperatively, active flexion-extension ankle motion and quadriceps strengthening exercises were encouraged. Partial weight-bearing with crutches as tolerated on the second postoperative day and full weight-bearing were allowed from the third day. For patients with THA, simultaneous flexion and internal rotation were avoided after surgery.

\section{Clinical evaluations and radiographic assessments}

Clinical follow-up was conducted routinely at 3 weeks, 3 months and 6 months after the procedures and annually thereafter until the final follow-up. The clinical evaluation protocol included the Harris Hip Score (HHS) [18], Knee Society Score (KSS) [19], and Short Form-12 scale (SF-12) [20]. Radiographs (serial standardized anteroposterior and lateral radiographs of hip and knee, and full-length weight-bearing anteroposterior films) were taken for patients preoperatively and performed at each follow-up. To minimize the variability of the interobserver, all radiographic measurements were performed independently and averaged by 2 trained investigators in the index surgery. To describe the coronal extremity axis, hip-knee-ankle (HKA) angle, femorotibial angle (FTA), femoral offset (FO), inclination angle, and limb length discrepancy (LLD) were measured and recorded. The radiological results are represented by the parameters, as shown in Fig. 1. Of note, when assessing coronal alignment on full-length films, an HKA less than $177^{\circ}$ was considered varus, neutral between $177^{\circ}$ and $183^{\circ}$, and valgus greater than $183^{\circ}$. Of note, severe valgus knee alignment was defined as an FTA of $<160^{\circ}$ [21].

Serial radiographs were also evaluated for evidence of postoperative periprosthetic fracture (PFF), dislocation, subsidence, and femoral component stability. Subsidence of the femoral component was defined as the change in the distance from the center of the femoral head to the lesser trochanter by the method of Heekin et al. [22]. The femoral component stability was evaluated and graded as bone stable, fibrous stable, or unstable, according to the criteria described by Engh et al. [23]. Complications included periprosthetic infection, deep venous thrombosis, and neurologic injury. Kaplan-Meier survivorship analysis was performed on all THAs and TKAs using a standard case scenario where all arthroplasties were considered to be successful at the final follow-up. Prosthesis failure was defined as any reason for aseptic revision.

\section{Statistical analysis}

All statistical analyses were performed using SPSS statistical software, version 25.0 (IBM Corp., Armonk, NY). The figures were generated by GraphPad Prism version 


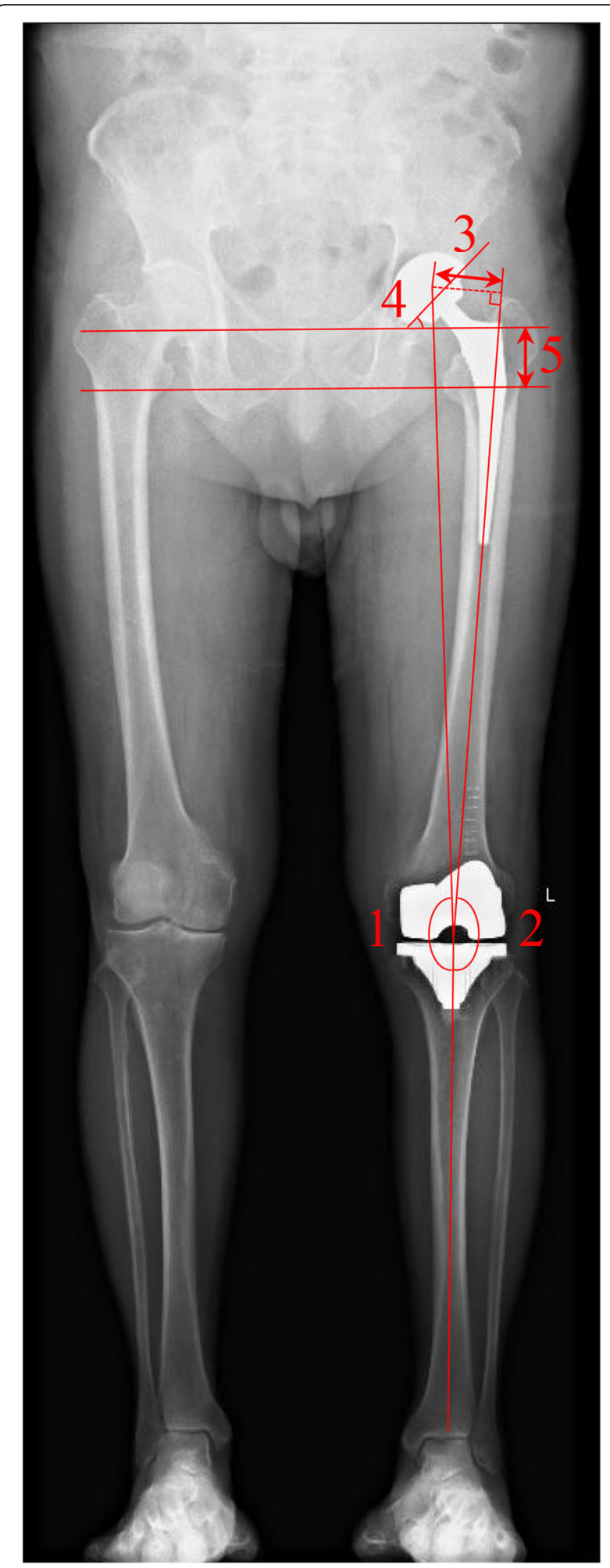

Fig. 1 Radiological angles. 1 Hip-knee-ankle angle (HKA). 2 Femorotibial angle (FTA). 3 Femoral offset (FO). 4 Cup inclination angle. 5 Limb length discrepancy (LLD)
8.0. The normal distribution of the data was tested using Kolmogorov-Smirnov's test. Depending on the distribution, categorical variables were compared using Fisher's exact test while continuous variables were evaluated with the student' t-test for normal distribution data or the Mann-Whitney U-test for skewness distribution data. Means and standard deviation for parametric or medians and $25-75 \%$ interquartile ranges for nonparametric data are present. Implant survivorships were analyzed by Kaplan-Meier curves with revision for any reason other than infection as the endpoint. The survival rate between the 2 groups was compared by the log-rank test. $\alpha=.05, P<.05$ indicated statistical significance. A post hoc power analysis was performed to determine the sample size necessary to distinguish differences in HSS and KSS at the follow-up intervals. According to the previous studies [24, 25], a 10-point difference in HSS or KSS was considered clinically significant. An alpha error probability and power were set to .05 and $80 \%$ using software (PASS, version 19.0), respectively. The required sample size was 33 patients for each group.

\section{Results}

The preoperative and postoperative clinical scores and radiological results are summarized in Table 2. No significant difference was detected in preoperative clinical parameters, including HHS, KSS, and SF-12. In both groups, the mean HHS, KSS, and SF-12 score significantly improved from preoperatively to the last followup $(p<.001)$. At the last clinical evaluation, the postoperative parameters, including HHS, KSS-knee score and function score, physical component summary (PCS) and mental component summary (MCS) of SF-12, were actually lower in the THA-TKA group. However, those differences were not significant $(p>.05)$.

There was also no significant difference in preoperative radiographic measurements between the 2 groups, including HKA, KAA, FO, and LLD (Table 2). However, preoperative mean HKA and FTA indicated valgus alignment in both groups $\left(184.1^{\circ}\right.$ vs $184.1^{\circ}, 173.0^{\circ}$ vs $173.2^{\circ}$, $p>.05)$. The comparisons of lower limb alignment parameters were based on the measured data of the fulllength weight-bearing radiographs, as shown in Figs. 2 and 3. Relative to the mechanical and anatomical alignment, HKA and FTA were corrected to neutral after surgery $(p<.001)$. The postoperative FO, LLD, and cup inclination did not differ between the 2 groups $(p=.967$, $.137, .323)$.

No periprosthetic infection or deep venous thrombosis was identified during the follow-up in each group (Table 3). There was 1 case of sciatic nerve palsy patient in the TKA-THA group after THA who recovered spontaneously within 9 months without residual symptoms. 
Table 2 Preoperative and postoperative clinical outcomes

\begin{tabular}{|c|c|c|c|}
\hline Parameters & $\begin{array}{l}\text { THA-TKA group } \\
n=28\end{array}$ & $\begin{array}{l}\text { TKA-THA group } \\
n=28\end{array}$ & $\begin{array}{l}P \\
\text { value }\end{array}$ \\
\hline \multicolumn{4}{|l|}{ Harris Hip Score } \\
\hline Preoperative & $34.0 \pm 4.3$ & $34.5 \pm 6.0$ & 0.722 \\
\hline Last follow-up & $82.2 \pm 6.4$ & $83.4 \pm 6.9$ & 0.496 \\
\hline \multicolumn{4}{|l|}{ Knee Society Score } \\
\hline Preoperative knee score & $32.4 \pm 6.4$ & $32.4 \pm 8.6$ & 0.986 \\
\hline Last follow-up knee score & $86.1 \pm 6.3$ & $86.4 \pm 8.4$ & 0.901 \\
\hline Preoperative function score & $38.0 \pm 6.0$ & $37.0 \pm 6.0$ & 0.506 \\
\hline Last follow-up function score & $85.0 \pm 4.3$ & $86.1 \pm 4.8$ & 0.382 \\
\hline \multicolumn{4}{|l|}{ SF-12 } \\
\hline Preoperative PCS & $10.5 \pm 2.4$ & $10.6 \pm 2.6$ & 0.831 \\
\hline Last follow-up PCS & $20.8 \pm 2.2$ & $21.0 \pm 2.1$ & 0.804 \\
\hline Preoperative MCS & $13.3 \pm 2.5$ & $13.5 \pm 2.9$ & 0.730 \\
\hline Last follow-up MCS & $22.9 \pm 1.8$ & $23.1 \pm 1.9$ & 0.519 \\
\hline \multicolumn{4}{|l|}{$\mathrm{HKA}\left({ }^{\circ}\right)$} \\
\hline Preoperative & $184.1 \pm 5.3$ & $184.1 \pm 5.5$ & 0.496 \\
\hline Last follow-up & $179.7 \pm 3.3$ & $179.6 \pm 2.4$ & 0.645 \\
\hline \multicolumn{4}{|l|}{ FTA $\left(^{\circ}\right)$} \\
\hline Preoperative & $173.0 \pm 5.5$ & $173.2 \pm 5.8$ & 0.600 \\
\hline Last follow-up & $177.7 \pm 3.2$ & $177.6 \pm 2.3$ & 0.178 \\
\hline \multicolumn{4}{|l|}{$\mathrm{FO}$} \\
\hline Preoperative & $39.0 \pm 9.0$ & $39.8 \pm 7.9$ & 0.705 \\
\hline Last follow-up & $40.5 \pm 6.5$ & $40.4 \pm 9.5$ & 0.967 \\
\hline \multicolumn{4}{|l|}{ LLD } \\
\hline Preoperative & $37.8 \pm 11.7$ & $41.2 \pm 8.1$ & 0.149 \\
\hline Last follow-up & $43.0 \pm 8.0$ & $46.5 \pm 9.1$ & 0.137 \\
\hline Cup inclination $\left(^{\circ}\right)$ & $41.9 \pm 5.9$ & $40.3 \pm 6.2$ & 0.323 \\
\hline
\end{tabular}

The values are present as the mean \pm SD

PCS Physical component summary, MCS Mental component summary, HKA Hip knee ankle angle, FTA Femorotibial angle, FO Femoral offset, LLD Limb length discrepancy

Intraoperative fracture occurred in 4 hips (1 in the distal femur and 3 in the proximal femur) and 1 knee without displaced cracks or perforation; all the fractures were treated with immediate cerclage wire fixation. Two patients (2 hips) experienced postoperative dislocation in the THA-TKA group who were treated with closed manipulative reduction and confined to bed for 4 weeks. Two patients in the THA-TKA group and 1 patient in the TKA-THA group had an over $5 \mathrm{~mm}$ subsidence of the femoral stem and underwent stem revisions with subsequent stabilization and evidence of fibrous stability at the final follow-up. Of the patients with aseptic revisions, in the THA-TKA group, two femoral revisions were performed for stem subsidence, and one acetabular revision was performed for recurrent dislocation. For ipsilateral TKA, two knee revisions were performed for tibial aseptic loosening, and 1 was performed for instability. In contrast, in the TKA-THA group, one femoral stem revision was performed for subsidence, one modular liner was changed because of dissociation of the highly cross-linked polyethylene insert from the outer shell, and one knee revision was performed due to tibial implant loosening. There was no difference between the THA-TKA group and the TKA-THA group with respect to the complication rate and overall revisions $(p>.05)$. Considering aseptic revision as an endpoint (Fig. 4), THA survival at 8 years was $94.7 \%(95 \%$ confidence interval $84.7-99.9 \%$ ) and $92.4 \%$ (95\% confidence interval 87.4-97.4\%) in the THA-TKA group and TKA-THA group, respectively (log-rank, $p=.939$ ). In contrast, the TKA survival at 8 years was $95.7 \%$ (95\% confidence interval $87.3-99.9 \%$ ) and $100.0 \%$ in the THA-TKA group and TKA-THA group, respectively (log-rank, $p=.187$ ). 


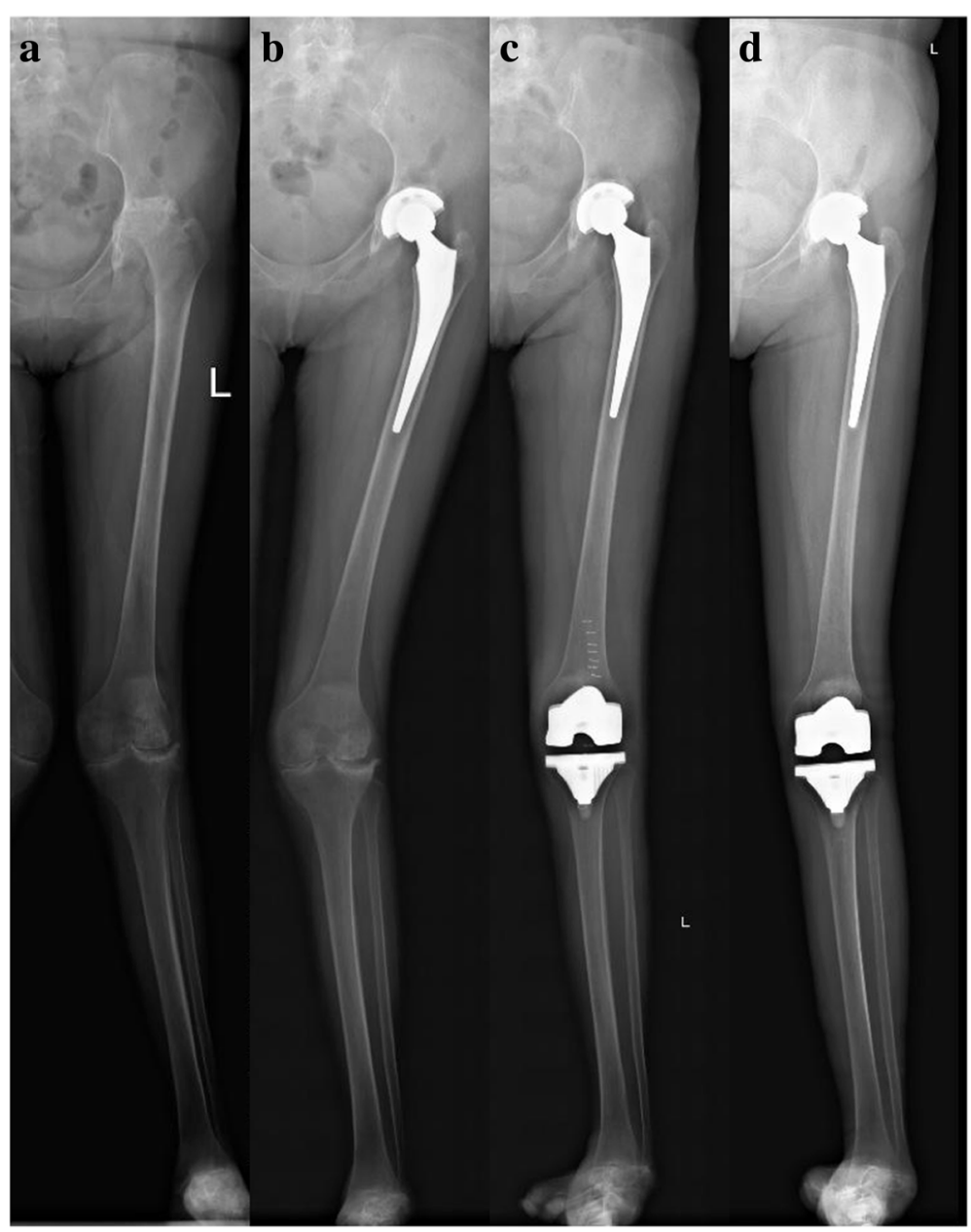

Fig. 2 A 43-year-old female with rheumatoid arthritis receiving TKA subsequent to ipsilateral THA. X-ray radiography at preoperative (a) and radiography at 3month follow-up after THA (b). Seven months after THA, the patient received ipsilateral TKA. The postoperative radiograph of the lower extremity (c) showed stable implant fixation. Postoperative radiographic image (d) at 7-year follow-up demonstrated that the acetabular, femoral, and tibial components were stable

\section{Discussion}

Previous studies demonstrated that biomechanical changes and loading redistribution after prior THA or TKA may affect the ipsilateral hip or knee [5-9]. However, no study has compared the clinical outcomes and implant survivorships in patients who underwent different sequences of ipsilateral THA and TKA. The most important finding of the present study was that there was no significant difference between the 2 groups in HHS, KSS, and SF-12 score at the final follow-up; confirming our hypothesis.

Only one study [12] assessed the influence of a prior THA on axial alignment and the clinical outcome of a subsequent ipsilateral TKA by comparing patients who underwent TKA followed by ipsilateral THA and those who only underwent TKA. Compared with the most comparable study by Asensio-Pascual et al. [12], the clinical scores from our results were lower than those reported in their study, with postoperative HHS, KSS-knee, and KSS- function scores of averaged 86.4, 87.6, 88.3, respectively in the THA-TKA group. A possible interpretation is that disease of our study, including RA, which is involved in multiple joint sites, including metatarsal phalangeal joints and the ankle, might be present even after TJA. The immobility of other lower limb joints could lead to difficulty ambulating or ascending stairs despite evident improvements in hip and knee function. If we compared the clinical scores in OA patients from our study with the results of Asensio-Pascual et al. [12], the mean postoperative HHS, KSS-knee, and KSS-function score was 82.6, 86.0, 85.0 in the THA-TKA group, respectively, which demonstrated similar results.

Comparison of the radiological results and complication rate with the only study [12] is difficult because it evaluated lower limb alignment without full-length weightbearing radiographs and did not systematically report the results of complications. Patients undergoing ipsilateral hip and knee surgeries presented preoperative valgus 


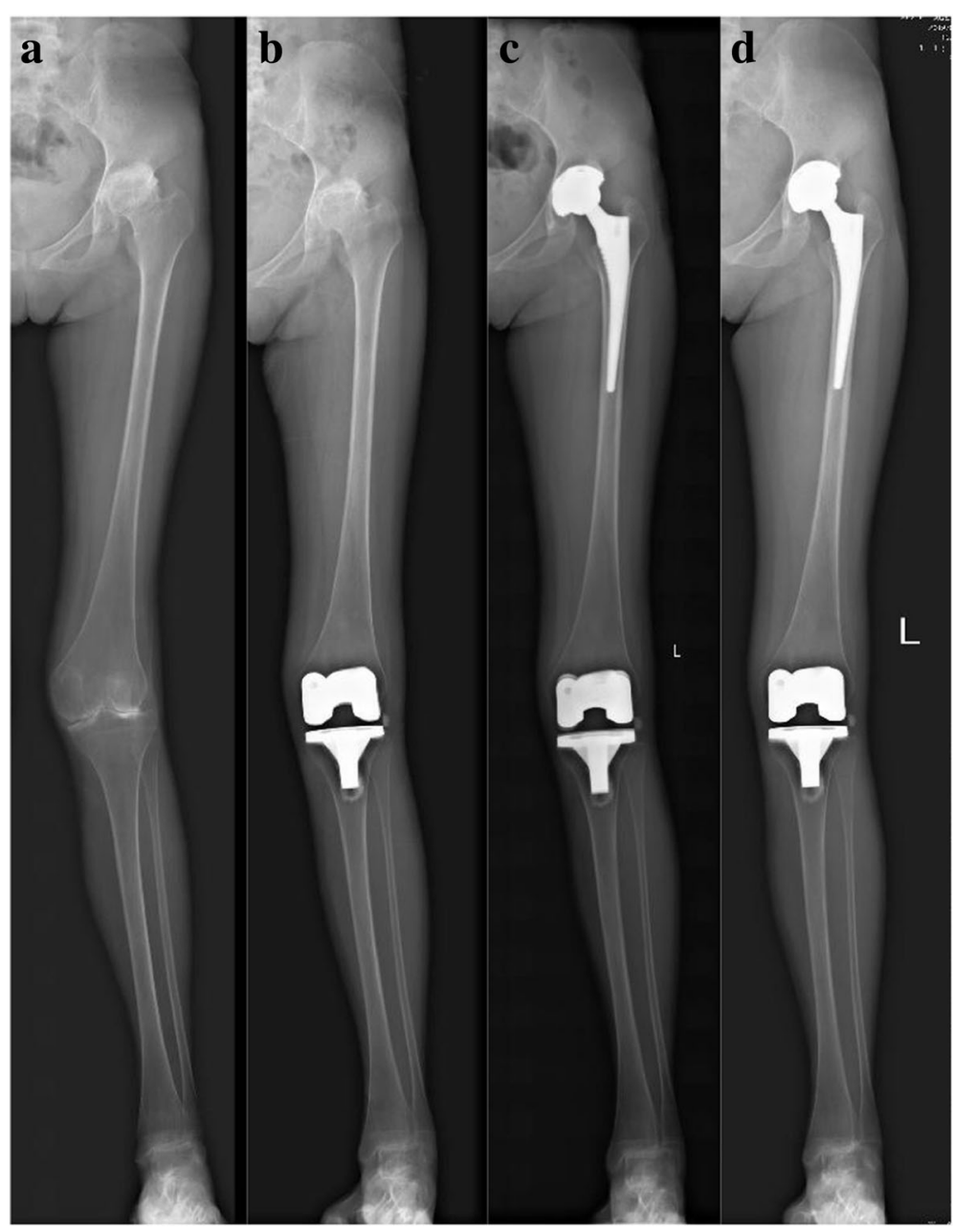

Fig. 3 Radiographs (a-d) of a 27-y-old woman who underwent THA subsequent to ipsilateral TKA for rheumatoid arthritis. a Preoperative lower extremity image. b Radiograph of the patient undergoing TKA at 6-month follow-up. One year after prior TKA, the patient underwent ipsilateral THA. c The radiograph of the lower extremity at 6-month follow-up after THA showed stable implant fixation. $\mathbf{d}$ Postoperative radiograph of acetabular, femoral, and tibial components at 8-year follow-up showed stable implant fixation

Table 3 Complications and revisions

\begin{tabular}{|c|c|c|c|}
\hline Complications & $\begin{array}{l}\text { THA-TKA group } \\
n=28\end{array}$ & $\begin{array}{l}\text { TKA-THA group } \\
n=28\end{array}$ & $\begin{array}{l}P \\
\text { value }\end{array}$ \\
\hline Postoperative PFF & 4 & 2 & 0.669 \\
\hline Early postoperative dislocation & 2 & 0 & 0.154 \\
\hline Femoral component stability & & & 0.429 \\
\hline Bony stable & 22 & 25 & \\
\hline Fibrous stable & 5 & 3 & \\
\hline Unstable & 1 & 0 & \\
\hline Femoral stem subsidence & 2 & 1 & 1.000 \\
\hline \multicolumn{4}{|l|}{ Revision } \\
\hline Total hip revision & 3 & 2 & 0.939 \\
\hline Total knee revision & 3 & 1 & 0.187 \\
\hline
\end{tabular}




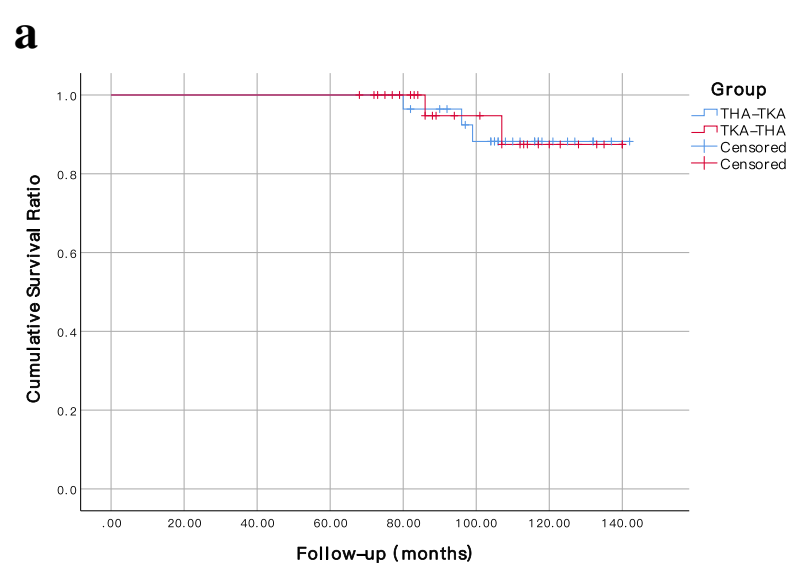

b

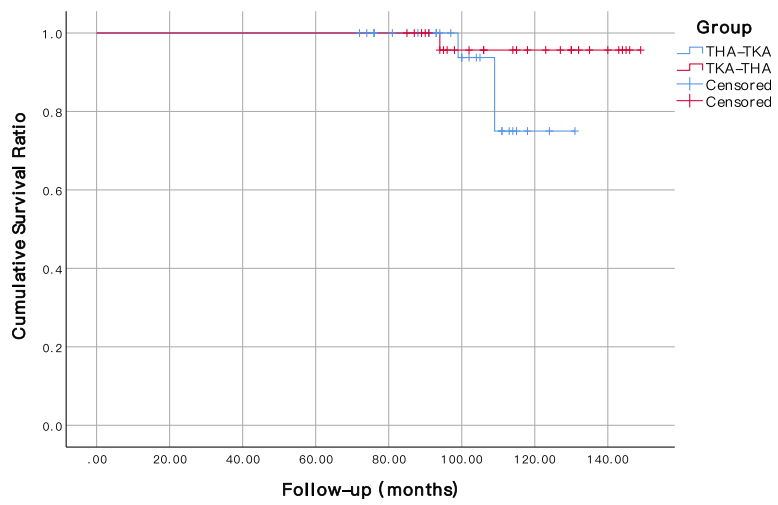

Fig. 4 Kaplan-Meier survival curve with revision of THA (a) and TKA (b) for any reason other than infection as the endpoint

deformity in the current study. Preoperative knee valgus deformity was associated with advanced RA, which was in agreement with a previous study [26]. Preoperative valgus deformity means that the procedure of TKA is much more technically challenging, including obtaining a proper component rotational alignment and balancing soft tissue in both flexion and extension with the least constraint. Although knees with preoperative valgus were corrected to neutral and no significant differences were detected between the 2 groups in axial alignment at the last followup, our overall implant survivorship of TKA in the THATKA group was lower than that reported in AsensioPascual et al. [12], with $96.6 \%$ survivorship at 7.2 years. A possible explanation could be that aseptic revision after TJA seems to be higher in patients with RA than tin hose with OA. Previous studies reported consistent outcomes to ours: a Dutch study [27] reported implant survival rates of $58 \%$ in RA patients, whereas the survival rate was $95 \%$ in OA patients in 18-year follow-up; Feng et al. [28] reported implant survival rates of $78.3 \%$ in RA patients compared to $92.7 \%$ in OA patients in 15-year follow-up; A meta-analysis study [29] reported that aseptic revision rates of $7.7 \%$ (46/594) in RA patients compared to $5.7 \%$ (52/904) in OA patients.

In clinical practice, if the ipsilateral hip and knee simultaneously meet the indications for TJA, most surgeons would suggest performing THA before TKA. Active flexion and extension of the knee depend largely on free hip function, and knee pain is always associated with ipsilateral hip dysfunction. However, certain affected multiple joint diseases, such as RA, normally erode the knees first, leading to compulsory TKA. Then, the disease severity of the ipsilateral hip gradually developed to a degree that met the indication for THA. We agreed with the idea that the sequence of arthroplasties should depend on the severity of symptoms and that the most symptomatic joint of the hip or knee should be replaced first $[8,30]$.
Although biomechanical changes and redistribution of loading of the lower limb were detected after THA or TKA in certain studies [5-9], the influence of a prior TJA on the subsequent ipsilateral THA or TKA is limited. Consistent with the findings of the current study, Foucher et al. [10] reported no increase in biomechanical loading during gait on the ipsilateral knee after THA. Likewise, other studies demonstrated that the changes in the axial alignment of the lower extremity after THA could result in an increased overload on the contralateral knee rather than ipsilateral knee, which was characterized by compensation to minimize the loading of the affected limb [7]. Thus, patients who undergo THA may have higher risks of developing OA in the contralateral knee than in the ipsilateral knee [31].

To date, this is the first study to compare the mid- to long-term results of patients who underwent different sequences of ipsilateral THA and TKA. The strengths of this study include the completeness of clinical and radiographic data, uncemented hip prostheses and posteriorstabilized knee prostheses, and the homogeneity of the surgical technique. However, we note that there are several limitations as well. Firstly, the retrospective design decreased the level of evidence and implied a selection and recall bias. Secondly, the small sample for each group may be insufficient power to detect difference in clinical and radiological outcomes. However, it was the largest sample-size study focused on the influence of different sequences of ipsilateral THA and TKA. Prospective data retrieval studies with larger samples are needed. Furthermore, we also recognize that our outcomes may suffer from bias due to different indications of the 2 diseases and different implants used in hip or knee arthroplasty. To minimize the possible bias of hip implants, case-control matching was performed for protheses of THA. Finally, only coronal alignment was evaluated in the present study, although the rotational alignment and 
patellar tilt are also important for the success of TKA. However, this study focused on modification of the mechanical axis, which is mainly present in coronal alignment. Future research with three-dimensional CT would be useful in studying modifications in rotational alignment.

\section{Conclusion}

Patients undergoing different ipsilateral THA and TKA sequences could achieve similar favorable clinical outcomes. Total joint arthroplasty can be performed safely with excellent outcomes in patients with a history of prior ipsilateral THA or TKA.

\author{
Abbreviations \\ TJA: Total joint arthroplasty; THA: Total hip arthroplasty; TKA: Total knee \\ arthroplasty; RA: Rheumatoid arthritis; OA: Osteoarthritis; HKA: Hip-knee- \\ angle; ITB: lliotibial band; LCL: Collateral ligament; POP: Popliteus tendon; \\ HHS: Harris Hip Score; KSS: Knee Society Score; SF-12: Short Form-12 Score; \\ FTA: Femorotibial angle; FO: Femoral offset; LLD: Limb length discrepancy; \\ PFF: Periprosthetic fracture; PCS: Physical component summary; MCS: Mental \\ component summary
}

\section{Acknowledgements}

We would like to thank the relevant staff for quidance and assistance for their support and collaboration in our hospital.

\section{Authors' contributions}

$\mathrm{ZH}-\mathrm{L}, \mathrm{WN}-\mathrm{Z}$, and $\mathrm{ZK}-\mathrm{Z}$ participated in the design and coordination of the study, collected the data, analyzed the data, and drafted the manuscript. ZY$\mathrm{L}, \mathrm{EZ}-\mathrm{Z}$, and $\mathrm{H}-\mathrm{L}$ assisted in collecting the data and drafting the manuscript. All authors have read and approved the final manuscript.

\section{Funding}

This research did not receive any specific grant from funding agencies in the public, commercial, or not-for-profit sectors.

\section{Availability of data and materials}

The datasets used or analyzed in the current study are available from the corresponding author up reasonable request.

\section{Declarations}

\section{Ethics approval and consent to participate}

This study was approved by the Ethics Committee and Institutional Review Board of West China Hospital, Sichuan University (2012-268). Written informed consent was obtained from each patient.

\section{Consent for publication}

All patients provided consent to participate in the current study, per our institution's standard for research consent forms. This consent specified that the research information obtained may be used for publication.

\section{Competing interests}

The corresponding author Dr. Zongke Zhou is a member of the editorial board of BMC Musculoskeletal Disorders, and the rest of the authors declare they have no competing interests.

Received: 2 August 2020 Accepted: 16 April 2021 Published online: 24 June 2021

\section{References}

1. Mertelsmann-Voss C, Lyman S, Pan TJ, Goodman SM, Figgie MP, Mandl LA. US trends in rates of arthroplasty for inflammatory arthritis including rheumatoid arthritis, juvenile idiopathic arthritis, and spondyloarthritis. Arthritis Rheumatol. 2014;66(6):1432-9. https://doi.org/10.1002/art.38384.
2. Ravi B, Croxford R, Reichmann WM, Losina E, Katz JN, Hawker GA. The changing demographics of total joint arthroplasty recipients in the United States and Ontario from 2001 to 2007. Best Pract Res Clin Rheumatol. 2012; 26(5):637-47. https://doi.org/10.1016/j.berh.2012.07.014.

3. Sanders TL, Maradit Kremers H, Schleck CD, Larson DR, Berry DJ. Subsequent total joint arthroplasty after primary total knee or hip arthroplasty: a 40-year population-based study. J Bone Joint Surg Am. 2017;99(5):396-401. https:// doi.org/10.2106/JBJS.16.00499.

4. Learmonth ID, Young C, Rorabeck C. The operation of the century: total hip replacement. Lancet. 2007;370(9597):1508-19. https://doi.org/10.1016/S01406736(07)60457-7.

5. Akiyama K, Nakata K, Kitada M, Yamamura M, Owaki H, Fuji T. Chronological changes in axial alignment of the ipsilateral hip and knee after total hip arthroplasty. J Arthroplast. 2018;33(2):415-22. https://doi.org/10.1016/j.arth.2 017.09.012.

6. Nam KW, Tsai TY, Dimitriou D, Li G, Kwon YM. Ipsilateral varus knee alignment correlates with increased femoral stem anteversion in primary total hip arthroplasty. Hip Int. 2016;26(2):175-9. https://doi.org/10.5301/hipint.5000325.

7. Akiyama K, Nakata K, Kitada $\mathrm{M}$, et al. Changes in axial alignment of the ipsilateral hip and knee after total hip arthroplasty. Bone Joint J. 2016;98b(3):349-58.

8. Stief F, Schmidt A, van Drongelen S, Lenarz K, Froemel D, Tarhan T, et al. Abnormal loading of the hip and knee joints in unilateral hip osteoarthritis persists two years after total hip replacement. J Orthop Res. 2018;36(8): 2167-77. https://doi.org/10.1002/jor.23886.

9. Wang W, Geller JA, Nyce JD, Choi JK, Macaulay W. Does ipsilateral knee pain improve after hip arthroplasty? Clin Orthop Relat Res. 2012;470(2):578-83. https://doi.org/10.1007/s11999-011-2116-3.

10. Foucher KC, Wimmer MA. Contralateral hip and knee gait biomechanics are unchanged by total hip replacement for unilateral hip osteoarthritis. Gait Posture. 2012;35(1):61-5. https://doi.org/10.1016/j.gaitpost.2011.08.006.

11. Webb BT, Ulrich SD, MacKinlay KGW, Smith L, Malkani A. Use of shorter intramedullary guide for ipsilateral total knee arthroplasty following prior total hip arthroplasty. J Knee Surg. 2018;31(4):348-51. https://doi.org/10.1 055/s-0037-1603796

12. Asensio-Pascual A, Lizaur-Utrilla A, Vizcaya-Moreno MF, et al. Prior unilateral total hip arthroplasty does not influence the outcome of ipsilateral total knee arthroplasty. Knee Surg Sports Traumatol Arthrosc. 2019;28(5):1452-7.

13. Alnahdi AH, Zeni JA, Snyder-Mackler L. Gait after unilateral total knee arthroplasty: frontal plane analysis. J Orthop Res. 2011;29(5):647-52. https:// doi.org/10.1002/jor.21323.

14. Milner CE, O'Bryan ME. Bilateral frontal plane mechanics after unilateral total knee arthroplasty. Arch Phys Med Rehabil. 2008;89(10):1965-9. https://doi. org/10.1016/j.apmr.2008.02.034.

15. Worsley P, Stokes M, Barrett D, et al. Joint loading asymmetries in knee replacement patients observed both pre- and six months post-operation. Clin Biomech (Bristol, Avon). 2013:28(8):892-7.

16. Agha R, Abdall-Razak A, Crossley E, Dowlut N, losifidis C, Mathew G, et al. STROCSS 2019 guideline: strengthening the reporting of cohort studies in surgery. Int J Surg. 2019;72:156-65. https://doi.org/10.1016/j.ijsu.2019.11.002.

17. Ranawat AS, Ranawat CS, Elkus M, et al. Total knee arthroplasty for severe valgus deformity. J Bone Joint Surg Am. 2005;87(Suppl 1 (Pt 2)):271-84.

18. Harris WH. Traumatic arthritis of the hip after dislocation and acetabular fractures: treatment by mold arthroplasty. An end-result study using a new method of result evaluation. J Bone Joint Surg Am. 1969;51(4):737-55. https://doi.org/10.2106/00004623-196951040-00012.

19. Insall JN, Dorr LD, Scott RD, et al. Rationale of the knee society clinical rating system. Clin Orthop Relat Res. 1989;(248):13-4.

20. Ware J Jr, Kosinski M, Keller SD. A 12-item short-form health survey: construction of scales and preliminary tests of reliability and validity. Med Care. 1996;34(3):220-33. https://doi.org/10.1097/00005650-199603 000-00003.

21. Liu HX, Shang $P$, Ying $X Z$, Zhang $Y$. Shorter survival rate in varus-aligned knees after total knee arthroplasty. Knee Surg Sports Traumatol Arthrosc 2016;24(8):2663-71. https://doi.org/10.1007/s00167-015-3781-7.

22. Heekin RD, Engh CA, Herzwurm PJ. Fractures through cystic lesions of the greater trochanter. A cause of late pain after cementless total hip arthroplasty. J Arthroplast. 1996:11(6):757-60. https://doi.org/10.1016/S0883-5403(96)80017-9.

23. Engh CA, Massin P, Suthers KE. Roentgenographic assessment of the biologic fixation of porous-surfaced femoral components. Clin Orthop Relat Res. 1990;(257):107-28 
24. Lee OS, Lee SH, Mok SJ, Lee YS. Comparison of the regeneration of cartilage and the clinical outcomes after the open wedge high tibial osteotomy with or without microfracture: a retrospective case control study. BMC Musculoskelet Disord. 2019:20(1):267. https://doi.org/10.1186/s12891-019-2607-z.

25. Jung WH, Takeuchi R, Chun CW, Lee JS, Jeong JH. Comparison of results of medial opening-wedge high tibial osteotomy with and without subchondral drilling. Arthroscopy. 2015;31(4):673-9. https://doi.org/10.1016/ j.arthro.2014.11.035.

26. Tucker A, O'Brien S, Doran E, et al. Total Knee arthroplasty in severe valgus deformity using a modified technique - a 10-year follow-up study. J Arthroplasty. 2019;34(1):40-46.e41.

27. van der Lugt JC, Onstenk R, Nelissen RG. Primary Stanmore total hip arthroplasty with increased cup loosening in rheumatoid patients. Int Orthop. 2003;27(5):291-3. https://doi.org/10.1007/s00264-003-0473-6.

28. Feng $B$, Weng $X$, Lin J, Jin J, Wang W, Qiu G. Long-term follow-up of cemented fixed-bearing total knee arthroplasty in a Chinese population: a survival analysis of more than 10 years. J Arthroplast. 2013;28(10):1701-6. https://doi.org/10.1016/j.arth.2013.03.009

29. Lee D-K, Kim H-J, Cho I-Y, Lee DH. Infection and revision rates following primary total knee arthroplasty in patients with rheumatoid arthritis versus osteoarthritis: a meta-analysis. Knee Surg Sports Traumatol Arthrosc. 2017; 25(12):3800-7. https://doi.org/10.1007/s00167-016-4306-8.

30. Strand V, Singh JA. Improved health-related quality of life with effective disease-modifying antirheumatic drugs: evidence from randomized controlled trials. Am J Manag Care. 2008;14(4):234-54.

31. Umeda N, Miki H, Nishii T, Yoshikawa H, Sugano N. Progression of osteoarthritis of the knee after unilateral total hip arthroplasty: minimum 10year follow-up study. Arch Orthop Trauma Surg. 2009;129(2):149-54. https:// doi.org/10.1007/s00402-008-0577-y.

\section{Publisher's Note}

Springer Nature remains neutral with regard to jurisdictional claims in published maps and institutional affiliations.

Ready to submit your research? Choose BMC and benefit from:

- fast, convenient online submission

- thorough peer review by experienced researchers in your field

- rapid publication on acceptance

- support for research data, including large and complex data types

- gold Open Access which fosters wider collaboration and increased citations

- maximum visibility for your research: over $100 \mathrm{M}$ website views per year

At $\mathrm{BMC}$, research is always in progress.

Learn more biomedcentral.com/submissions 